\title{
Clinicopathological characteristics of thyroid cancer in the federal state of Salzburg
}

\author{
Gundula Rendl · Margarida Rodrigues $(\mathbb{D}) \cdot$ Gregor Schweighofer-Zwink - Josef Hutter · Anton Hittmair · \\ Barbara Zellinger · Cornelia Hauser-Kronberger · Christian Pirich
}

Received: 24 March 2016 / Accepted: 12 April 2017 / Published online: 10 May 2017

(C) The Author(s) 2017. This article is an open access publication.

\begin{abstract}
Summary
Objective The aim of our investigation was to evaluate the clinicopathological characteristics and mutation patterns in newly diagnosed cases of thyroid cancer in the federal state of Salzburg, Austria, in the year 2013. Methods The medical records of all patients newly diagnosed with thyroid cancer in 2013 in the federal state of Salzburg were retrospectively reviewed. The clinicopathological characteristics and mutations of thyroid cancers were analyzed.

Results 63 patients (mean age: 51.0 years, range: 21-81 years; female $75 \%$, male $25 \%$ ) were identified. 53 patients had papillary (12 follicular variant), 4 patients follicular (1 oxyphilic variant), 3 patients medullary, and 3 patients anaplastic thyroid cancer. T1 tumors were found in 34 patients (pTla, 20 patients; pTlb, 14 patients), T2 tumors in 10 patients, $\mathrm{T} 3$ tumors in 16 patients, and T4 tumors in 3 patients. Lymph node involvement was seen in 15 patients and metastatic disease in 1 patient. Mutations of BRAF (Btype Raf kinase) were detected in 23 and mutation of NRAS (Neuroblastoma RAS Viral Oncogene Homolog)
\end{abstract}

\footnotetext{
G. Rendl · M. Rodrigues · G. Schweighofer-Zwink · Prof. C. Pirich, MD ( $\triangle)$

Department of Nuclear Medicine and Endocrinology, Paracelsus Medical University Salzburg, Müllner Hauptstrasse 48, 5020 Salzburg, Austria c.pirich@salk.at

\section{J. Hutter}

Department of Nuclear Medicine, Schwarzach Hospital, Schwarzach, Austria
}

\author{
A. Hittmair \\ Department of Pathology, Schwarzach Hospital, \\ Schwarzach, Austria
}

B. Zellinger · C. Hauser-Kronberger

Department of Pathology, Paracelsus Medical University Salzburg, Salzburg, Austria in 2 papillary thyroid cancers. No concomitant mutations of BRAF and NRAS were found.

Conclusion Females accounted for $75 \%$ of the patients with newly diagnosed thyroid cancer and the incidence peaked at a younger age than in males. Papillary thyroid cancer was the most frequent tumor type, accounting for $84 \%$ of the cases. A high frequency of T1 tumors and cancers with no lymph node involvement was found. Males had a higher proportion of large tumors and more aggressive forms of thyroid cancer than females. Mutations (mostly of BRAF) were found in $47 \%$ of the cases. Neither mutations of KRAS (Kirsten rat sarcoma viral oncogene homologue) nor concomitant mutations of BRAF and NRAS were found.

Keywords Thyroid cancer · Endocrine system · Diagnosis - Gene mutation · Fine-needle aspiration biopsy

\section{Introduction}

Thyroid cancer is the most frequent cancer among endocrine tumors. Its incidence has been increasing over the past several decades worldwide [1-3], showing considerable regional variation [4-9].

The increased incidence of thyroid cancer is often attributed to increased detection associated with better access to healthcare, diligent health screening with rising use of thyroid imaging, and optimized management strategies [1, 3, 8-10]. Small papillary thyroid cancers, including papillary microcarcinomas, have mainly contributed to the worldwide increased incidence of thyroid cancer observed over the past decades $[1,3,5,7]$. However, the increasing number of large tumors and rates of thyroid cancer-related mortality in spite of earlier treatment, as well as the changes in thyroid cancer molecular profiles, also suggest a true increase in the number 
of cases. Hormonal influences, including increased iodine intake and chronic lymphocytic thyroiditis, and recently increased and thyroid-specific environmental carcinogens, including exposure to radiation and environmental pollutants such as nitrates, heavy metals, and other compounds largely used in the industrialized society [3,11-13], could be responsible. Causal relationships require further experimental and epidemiological studies.

Thyroid cancer exists in several forms. Differentiated thyroid cancers include papillary and follicular thyroid cancer, and both arise from the thyroid follicular cells. Medullary thyroid cancer arises from the parafollicular cells. Anaplastic thyroid cancer is one of the most aggressive and rapidly fatal cancers. It can develop from differentiated thyroid cancer that de-differentiates over time, or can also arise de novo [14]. The prognosis and treatment of thyroid cancer depend on the tumor type and its stage at the time of diagnosis [13, 15]. Age, the completeness of resection, and extrathyroidal extension are prognostic indicators employed in many staging systems for differentiated thyroid cancer [13-15].

Fine-needle aspiration biopsy (FNAB) is the gold standard for the differential diagnosis of thyroid nodules, but has the limitation of inadequate sampling or indeterminate lesions. Molecular analysis of gene mutations was found to improve the diagnostic performance of FNAB [16].

The aim of the present investigation was twofold: first, to assess the clinicopathological characteristics of newly diagnosed thyroid cancers in the federal state of Salzburg, Austria, in the year 2013; and secondly, to analyze mutations in differentiated thyroid cancers. Furthermore, the differences in clinical routine leading to the first diagnosis of thyroid cancer in two endocrine centers were evaluated.

\section{Materials and methods}

The medical records of all patients who were newly diagnosed with thyroid cancer (ICD10 code C73) during the year 2013 in the federal state of Salzburg were retrospectively reviewed. We compared the differences in clinical routine leading to thyroid gland surgery and diagnosis of thyroid cancer between two endocrine centers in the federal state of Salzburg.

The clinicopathological characteristics of all patients with thyroid cancer and gene mutation tests (performed for BRAF [B-type Raf kinase], KRAS [V-Kiras2 Kirsten rat sarcoma viral oncogene homolog], and NRAS [Neuroblastoma RAS Viral Oncogene Homolog]) in differentiated thyroid cancers were evaluated.

\section{Results}

\section{Clinicopathological characteristics}

A total of 63 patients with newly diagnosed thyroid cancer were identified retrospectively (cancer registry) in the federal state of Salzburg in 2013. 47 patients were female $(75 \%)$ and 16 patients were male $(25 \%)$. The mean age at diagnosis was 51.0 years (female, 48.3 years; male, 51.9 years; range: $21-81$ years).

The differences in clinical routine in the two centers were that in center 1 , patients with hypofunctional thyroid nodules or otherwise morphologically suspicious thyroid nodules underwent preoperative ultrasound-guided FNAB. In center 2, hypofunctional thyroid nodules or morphologically suspicious thyroid nodules only underwent preoperative FNAB, which was not ultrasound-guided, if the nodule was palpable.

In center 1,33 patients ( $52 \%$ ) were newly diagnosed with thyroid cancer in 2013. Out of these, 32 patients underwent thyroid surgery because of the following findings: 23 patients had suspicious thyroid nodules (20 patients underwent preoperative FNAB, which was positive for thyroid cancer in 17 patients and inconclusive in 3 patients), 8 patients showed growing nodules in multinodular goiter, and 1 patient had concomitant Graves' and multinodular disease. The remaining 1 patient was not operated upon due to the diagnosis of anaplastic thyroid carcinoma.

In center 2,30 patients (48\%) were newly diagnosed with thyroid cancer in 2013. 28 of these patients underwent thyroid surgery due to the following findings: 25 patients had suspicious thyroid nodules (15 patients underwent preoperative FNAB, which was positive for thyroid cancer in 14 patients and inconclusive in 1 patient), 2 patients showed growing nodules in multinodular goiter, and 1 patient had recidivated Graves' disease. The other 2 patients were not operated upon because of the diagnosis of anaplastic thyroid carcinoma.

Overall, 60 patients (95\%) underwent thyroid surgery. Preoperative FNAB was applied in 38 patients $(60 \%)$. It was positive for thyroid cancer in 34 patients (90\%) and inconclusive in 4 patients (10\%).

The histological types were papillary thyroid cancer in 53 patients (84\%; follicular variant in 12 patients, 19\%), follicular thyroid cancer in 4 patients (6\%; oxyphilic variant in 1 patient, $2 \%$ ), medullary thyroid cancer (all sporadic in genetic analysis for MEN IIa/IIb) in 3 patients (5\%), and anaplastic thyroid cancer in 3 patients (5\%). The tumors were unifocal in 50 patients (79\%) and unilateral in 52 patients (83\%).

T1 tumors were found in 34 patients (54\%). Of these patients, 20 patients $(32 \%)$ had a pTla tumor and 14 patients $(22 \%)$ had a pT1b tumor. T2 tumors were diagnosed in 10 patients $(16 \%)$, T3 tumors in 16 patients $(25 \%)$, and T4 tumors in 3 patients $(5 \%)$. 
Table 1 Distribution of T-stage and N-stage

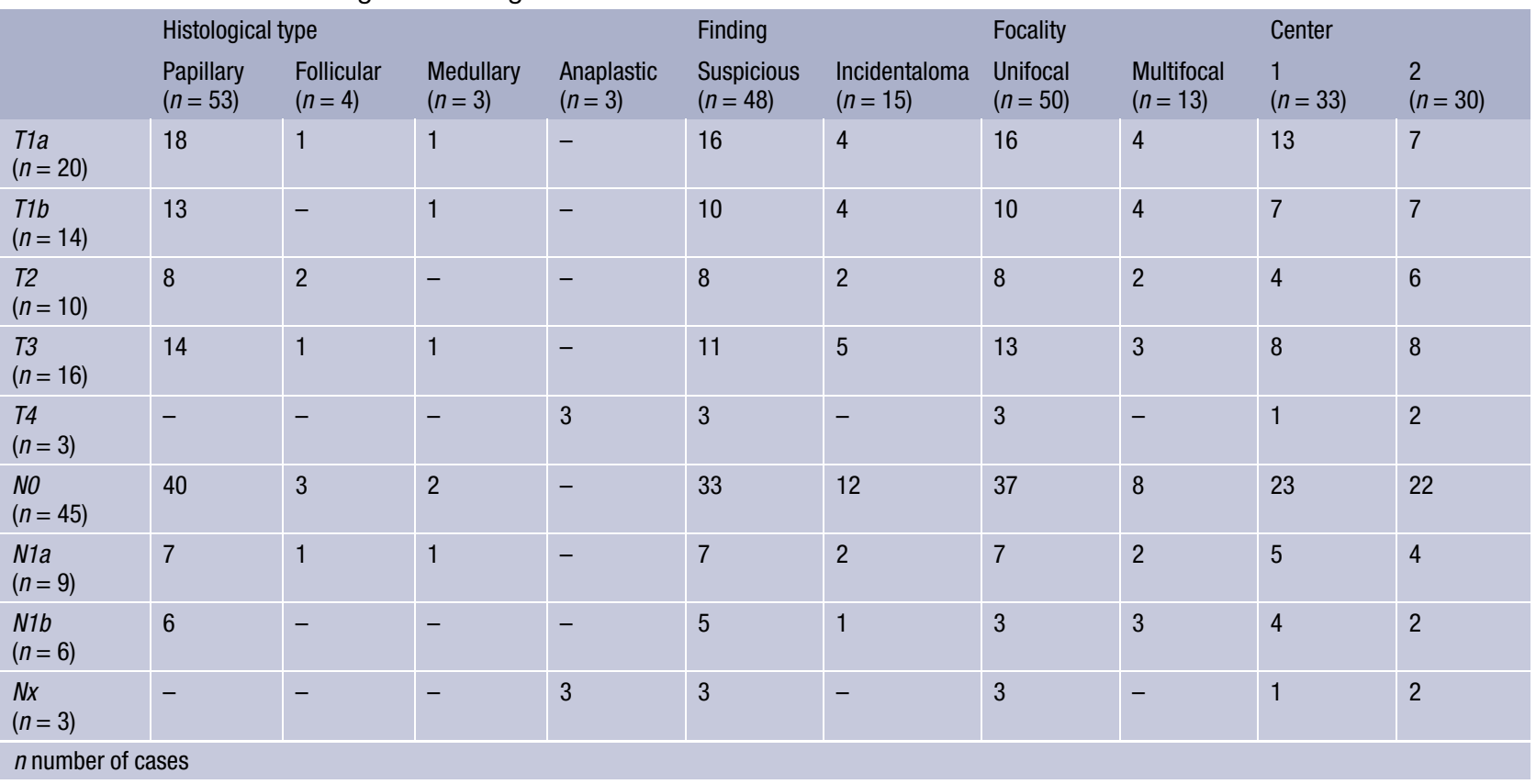
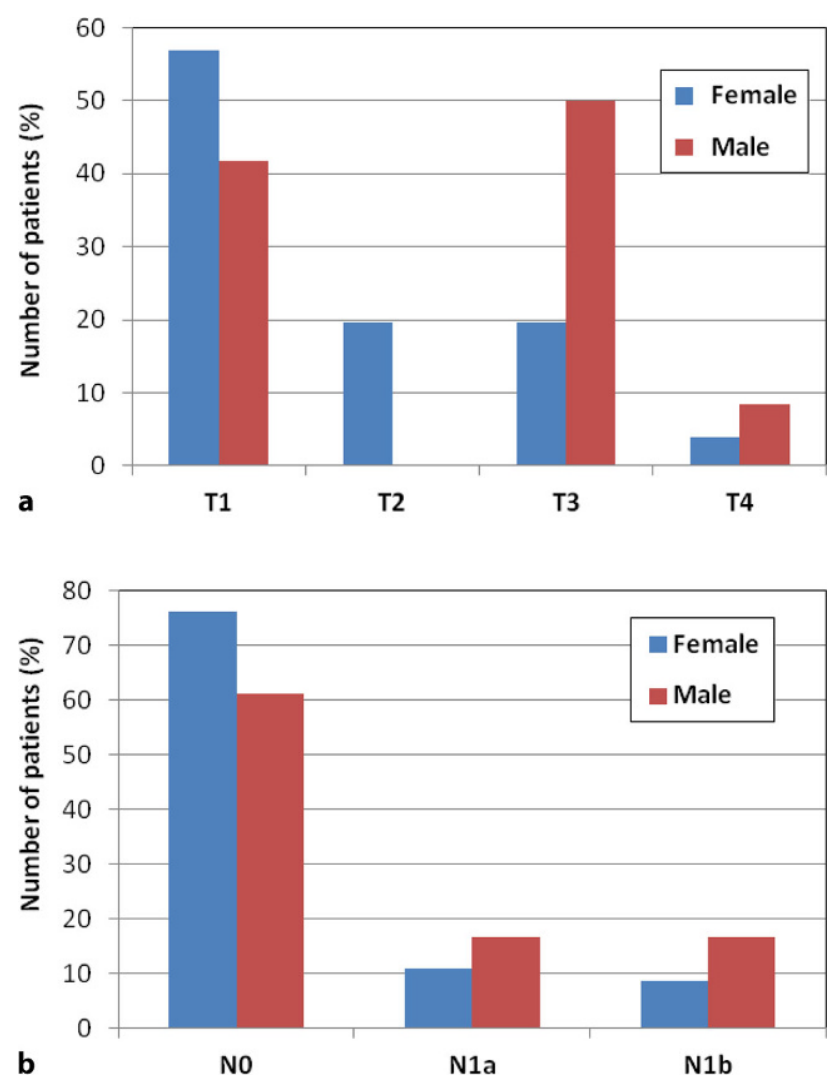

Fig. 1 Distribution of $\mathbf{a} \mathrm{T}$-and $\mathbf{b} \mathrm{N}$-stage across gender

Lymph node involvement was seen in 15 patients (24\%) and metastatic disease (pulmonary metastases) in 1 patient $(2 \%)$ with papillary thyroid cancer (pT3 N0 M1). Table 1 shows the distribution of T-stage and $\mathrm{N}$-stage across histological type, finding, focality, and center. Fig. 1 illustrates the distribution of T-stage and N-stage across gender. Female patients with all histological types peaked at a younger age than male patients, except patients with medullary thyroid cancer. Local and vascular invasion were found in 7 (11\%) and 5 patients $(8 \%)$, respectively.

\section{Gene mutation analysis}

Mutations of BRAF, KRAS, and NRAS were tested in tissue samples of 53 patients (39 papillary thyroid cancers, 10 with follicular variant; 3 follicular thyroid cancers, 1 oxyphilic variant).

BRAF mutations were detected in 23 cases with papillary thyroid cancer $(43 \%)$. NRAS mutations (in codon 61) were found in 2 other cases with papillary thyroid cancer $(4 \%$; 1 case with follicular variant).

No case was found with overlapping mutation of BRAF and NRAS.

No mutations of BRAF and NRAS were found in 28 cases $(53 \%$; 16 patients with papillary thyroid cancer, 8 patients with follicular variant; 3 patients with follicular thyroid cancer, 1 patient with oxyphilic variant). Table 2 shows the distribution of BRAF and NRAS mutations across $\mathrm{T}$-stage and $\mathrm{N}$-stage.

None of the 53 cases investigated showed a KRAS mutation.

No correlation between gene mutations and gender, age, or TNM classification of tumors was found.

\section{Discussion}

In Austria, despite the introduction of iodine goiter prophylaxis in the early sixties, multinodular goiter remains endemic [17]. Both gender distribution and 
Table 2 Distribution of BRAF and NRAS mutations across T-stage and $\mathrm{N}$-stage

\begin{tabular}{|l|l|l|l|l|}
\hline & $\begin{array}{l}\text { BRAF } \\
\text { BRAF } \\
\text { mutations } \\
(n=23)\end{array}$ & $\begin{array}{l}\text { No BRAF } \\
\text { mutation } \\
(n=30)\end{array}$ & $\begin{array}{l}\text { NRAS } \\
\text { NRAS } \\
\text { mutation } \\
(n=2)\end{array}$ & $\begin{array}{l}\text { No NRAS } \\
\text { mutation } \\
(n=51)\end{array}$ \\
\hline $\begin{array}{l}\text { T1a } \\
(n=18)\end{array}$ & 11 & 7 & - & 18 \\
\hline $\begin{array}{l}T 1 b \\
(n=13)\end{array}$ & 6 & 7 & 1 & 12 \\
\hline $\begin{array}{l}T 2 \\
(n=9)\end{array}$ & 2 & 7 & 1 & 8 \\
\hline $\begin{array}{l}T 3 \\
(n=13)\end{array}$ & 4 & 9 & - & 13 \\
\hline $\begin{array}{l}\text { NO } \\
(n=39)\end{array}$ & 16 & 23 & 2 & 37 \\
\hline $\begin{array}{l}\text { N1a } \\
(n=8)\end{array}$ & 5 & 3 & - & 8 \\
\hline $\begin{array}{l}\text { N1b } \\
(n=6)\end{array}$ & 2 & 4 & - & 6 \\
\hline $\begin{array}{l}n \text { number of cases, BRAFB-type Raf kinase, } \\
\text { Oncogene Homolog Neuroblastoma RAS Viral }\end{array}$
\end{tabular}

peak age at diagnosis of thyroid cancer in the federal state of Salzburg are similar to those reported in the literature $[1,3,5,8]$. In our population the proportion of $\mathrm{T} 1$ tumors and cancers with no lymph node involvement was high (54 and $76 \%$, respectively). These favorable pathological characteristics may be attributable to the wide use of imaging studies-including ultrasound, computed tomography, magnetic resonance imaging, and positron-emission tomography scans - that incidentally detect thyroid nodules, as also found by other investigators $[9,10]$. Furthermore, FNAB (which was inconclusive in only $10 \%$ of this series of patients) makes an important contribution to decision making in the management of suspicious thyroid nodules and earlier diagnosis of thyroid cancer. Consistently, the proportion of $\mathrm{T} 1$ tumors found in center 1 (where all patients with hypofunctional thyroid nodules or otherwise morphologically suspicious thyroid nodules underwent preoperative ultrasound-guided FNAB) was higher than that seen in center 2. However, it is noteworthy that in our series, both the proportion of T3 and T4 tumors, and the numbers of cancers with extrathyroidal invasion were higher in male than in female patients. These pathological differences found between female and male patients suggest that other influences besides optimized diagnosis could be responsible for the increased incidence of thyroid cancer.

Local lymph node invasion was found in $24 \%$ of patients of our series. These results support the further use of single-photon-emission computed tomography (SPECT)/computed tomography (CT) in the follow-up of thyroid cancer patients.

In our study population, the proportion of patients with papillary thyroid cancers, in particular with $\mathrm{T} 1$ tumors, was high. This finding is consistent with the reports of a prominent increase in small, papillary thy- roid cancers in several regions $[1,3,5,7,18]$. This might be associated with a high iodine intake, which is reported to be related to the development of papillary thyroid cancers [1, 19, 20]. Neuhold et al. [17] suggested that the levels of iodine intake play only a minor role in the early phase of carcinogenesis of papillary thyroid cancer, but may be of some importance during the progression of latent to clinically evident papillary thyroid cancer. However, the association between thyroid cancer and iodine intake remains unclear.

Thyroid cancers have been found to harbor multiple gene mutations or rearrangements [16, 21-25]. Cantara et al. [16] reported that the presence of mutations at cytology was associated with cancer $91 \%$ of times and follicular adenoma 9\% of times. BRAF mutations were always associated with cancer [16], whereas RAS mutations were mainly associated with cancer $(74-88 \%)[16,21]$, but also with follicular adenoma (26\%) [16]. RAS-mutated thyroid cancers were found to be prone to distant metastases in lung and bone, rather than to locoregional lymph node involvement [23]. In our series, mutations in thyroid tissue samples were found in $47 \%$ of cases, all with papillary thyroid cancer. Of these mutations, $92 \%$ were of BRAF and $8 \%$ were of NRAS. These data are in agreement with findings that BRAF is the most common gene mutation (detected in approximately $50 \%$ of sporadic papillary thyroid cancers) [24, 25] and NRAS mutations (found with a frequency of $6 \%$ by Fukushima et al) [22] are the most prevalently identified additional mutations [21, 22, 24-26]. RET/PTC rearrangement, BRAF, NRAS, and KRAS mutations are considered to be mutually exclusive in papillary thyroid carcinoma [23, 24]. However, Zou et al. [27] reported recently that concomitant mutations of RET/PTC, RAS, or BRAF are a frequent event in advanced papillary thyroid carcinoma and are associated with poor prognosis. The concomitant mutations may represent intratumor heterogeneity and could exert a gene dosage effect to promote disease progression. Of note, we found no case with mutation of KRAS, or with simultaneous mutation of BRAF and NRAS. These findings might also be associated with earlier diagnosis of thyroid cancer in our series. Further studies on the value of preoperative mutation analysis for determining the possibility of malignancy, the occurrence of simultaneous gene mutations in thyroid cancer, and their significance for tumor progression and survival are warranted.

In conclusion, in the federal state of Salzburg, females accounted for $75 \%$ of the patients with thyroid cancer and rates peaked at a younger age than in male patients (except for medullary thyroid cancer). The main indication for surgery was a suspicious thyroid nodule. Papillary thyroid cancer was the most frequent tumor type, accounting for $84 \%$ of the cases. High frequencies of T1 tumors and cancers with no lymph node involvement were found, espe- 
cially for papillary thyroid cancer. Male patients had a higher proportion of large tumors and more aggressive forms of thyroid cancer than female patients. Mutations were seen in $47 \%$ of the cases and BRAF was the most common mutation. Neither mutations of KRAS nor concomitant mutations of BRAF and NRAS were found.

Open access funding provided by Paracelsus Medical University Salzburg.

\section{Compliance with ethical guidelines}

Conflict of interest G. Rendl, M. Rodrigues, G. SchweighoferZwink, J. Hutter, A. Hittmair, B. Zellinger, C. Hauser-Kronberger, and C. Pirich declare that they have no competing interests.

Ethical standards All procedures performed in studies involving human participants were in accordance with the ethical standards of the institutional and/or national research committee and with the 1964 Helsinki declaration and its later amendments or comparable ethical standards. Informed consent was obtained from all individual participants included in the study.

Open Access This article is distributed under the terms of the Creative Commons Attribution 4.0 International License (http://creativecommons.org/licenses/by/4.0/), which permits unrestricted use, distribution, and reproduction in any medium, provided you give appropriate credit to the original author(s) and the source, provide a link to the Creative Commons license, and indicate if changes were made.

\section{References}

1. Cho BY, Choi HS, ParkYJ, et al. Changes in the clinicopathological characteristics and outcomes of thyroid cancer in Korea over the pastfour decades. Thyroid. 2013;23:797-804.

2. Nguyen QT, Lee EJ, Huang MG, et al. Diagnosis and treatment of patients with thyroid cancer. Am Health Drug Benefits. 2015;8:30-40.

3. Vigneri R, Malandrino P, Vigneri P. The changing epidemiology of thyroid cancer: Why is incidence increasing? Curr Opin Oncol. 2015;27:1-7.

4. Aschebrook-Kilfoy B, Ward MH, Sabra MM, et al. Thyroid cancer incidence patterns in the United States by histologic type, 1992-2006. Thyroid. 2011;21:125-34.

5. Elisei R, Molinaro E, Agate L, et al. Are the clinical and pathological features of differentiated thyroid carcinoma really changed over the last 35 years? Study on 4187 patients from a single Italian institution to answer this question. JClin Endocrinol Metab. 2010;95:1516-27.

6. Kilfoy BA, Zheng T, Holford TR, et al. International patterns and trends in thyroid cancer incidence, 1973-2002. Cancer Causes Control. 2009;20:525-31.

7. Machens A, Dralle H. Decreasing tumor size of thyroid cancer in Germany: institutional experience 1995-2009. EurJEndocrinol. 2010;163:111-9.

8. Olaleye O, Ekrikpo U, MoorthyR, et al. Increasing incidence of differentiated thyroid cancer in South East England: 1987-2006. Eur Arch Otorhinolaryngol. 2010;268:899-906.
9. Van den Bruel A, Francart J, Dubois C, et al. Regional variation in thyroid cancer incidence in Belgium is associated with variation in thyroid imaging and thyroid disease management. JClin Endocrinol Metab. 2013;98:4063-71.

10. Zevallos JP, Hartman CM, Kramer JR, etal. Increased thyroid cancer incidence corresponds to increased use of thyroid ultrasound and fine-needle aspiration: a study of the Veterans Affairs health care system. Cancer. 2015;121:741-6.

11. Hayashi Y, Lagarde F, Tsuda N, et al. Papillary microcarcinoma of the thyroid among atomic bomb survivors: tumor characteristics and radiation risk. Cancer. 2010;116:1646-55.

12. Rego-Iraeta A, Perez-Mendez LF, Mantinan B, et al. Time trends for thyroid cancer in northwestern Spain: true rise in the incidence of micro and larger forms of papillary thyroid carcinoma. Thyroid. 2009;19:333-40.

13. Schneider DF, Chen H. New developments in the diagnosis and treatment of thyroid cancer. CA Cancer J Clin. 2013;63:373-94.

14. Patel KN, Shaha AR. Poorly differentiated and anaplastic thyroid cancer. Cancer Control. 2006;13:119-28.

15. Zarebczan B, Chen H. Multi-targeted approach in the treatment of thyroid cancer. Minerva Chir. 2010;65:59-69.

16. Cantara S, Capezzone M, Marchisotta S, et al. Impact of proto-oncogene mutation detection in cytological specimens from thyroid nodules improves the diagnostic accuracy of cytology. JClin Endocrinol Metab. 2010;95:1365-9.

17. Neuhold N, Kaiser H, Kaserer K. Latent carcinoma of the thyroid in Austria: a systematic autopsy study. Endocr Pathol. 2001;12:23-31.

18. Asioli S, Odasso C, Macrì L, Palestini N, Bussolati G. Merits of the PMiT (papillary microtumor) terminology in the definition of a subset of incidental papillary microcarcinomas of the thyroid. Int J Surg Pathol. 2009;17:378-83.

19. Blomberg M, Feldt-Rasmussen U, Andersen K, Kjaer S. Thyroid cancer in Denmark 1943-2008, before and after iodine supplementation. Int JCancer. 2012;131:2360-6.

20. Pettersson B, Coleman MP, Ron E, Adami HO. Iodine supplementation in Sweden and regional trends in thyroid cancer incidence by histopathologic type. Int J Cancer. 1996;65:13-9.

21. Bhaijee F, Nikiforov YE. Molecular analysis of thyroid tumors. Endocr Pathol. 2011;22:126-33.

22. Fukushima T, Suzuki S, Mashiko M, et al. BRAF mutations in papillary carcinomas of the thyroid. Oncogene. 2003;22:6455-7.

23. Kimura ET, Nikiforova MN, Zhu Z, Knauf JA, Nikiforov YE, Fagin JA. High prevalence of BRAF mutations in thyroid cancer: genetic evidence for constitutive activation of the RET/PTC-RAS-BRAF signaling pathwayin papillary thyroid carcinoma. Cancer Res. 2003;63:1454-7.

24. Park SJ, Sun JY, Hong K, et al. Application of BRAF, NRAS, KRAS mutations as markers for the detection of papillary thyroid cancer from FNAB specimens by pyrosequencing analysis. Clin Chem Lab Med. 2013;51:1673-80.

25. Tobiás B, Halászlaki C, Balla B, et al. Genetic alterations in Hungarian patients with papillary thyroid cancer. Pathol OncolRes. 2016;22:27-33.

26. Eszlinger M, Neustadt M, Ruschenburg I, et al. Fineneedle aspiration cytology of thyroid nodules: molecular diagnostics in a routine diagnostic setting. Dtsch Med Wochenschr. 2014;139:476-80.

27. Zou M, Baitei EY, Alzahrani AS, et al. Concomitant RAS, RET/PTC, or BRAF mutations in advanced stage of papillary thyroid carcinoma. Thyroid. 2014;24:1256-66. 